\title{
In-Hospital Mortality of Disseminated Tuberculosis in Patients Infected with the Human Immunodeficiency Virus
}

\author{
Rodrigo Pires dos Santos, ${ }^{1,2}$ Caroline Deutschendorf, ${ }^{1}$ Karin Scheid, ${ }^{1}$ \\ and Luciano Zubaran Goldani ${ }^{1}$
${ }^{1}$ Infectious Disease Service, Hospital de Clínicas de Porto Alegre (HCPA), Universidade Federal do Rio Grande do Sul (UFRGS), 90035-003, Porto Alegre, RS, Brazil
${ }^{2}$ Comissão de Controle de Infecção, Hospital de Clínicas de Porto Alegre (HCPA), 90035-003 Ramiro Barcelos 2350, Porto Alegre, RS, Brazil

Correspondence should be addressed to Rodrigo Pires dos Santos, rpsantos@hcpa.ufrgs.br

Received 13 April 2010; Accepted 2 July 2010

Academic Editor: Graeme Ayton Meintjes

Copyright ( 2011 Rodrigo Pires dos Santos et al. This is an open access article distributed under the Creative Commons Attribution License, which permits unrestricted use, distribution, and reproduction in any medium, provided the original work is properly cited.

\begin{abstract}
Background. Tuberculosis (TB) is a cause of significant morbidity and mortality in patients with AIDS. The goal of our study was to determine predictors of in-hospital mortality in patients with AIDS and disseminated tuberculosis in a middle-income country. Material and Methods. We conducted a retrospective cohort study in a tertiary care center, for patients with AIDS in southern Brazil. From 1996 to 2008, all patients with the diagnosis of disseminated TB were included. Results. Eighty patients were included. In-hospital mortality was 35\% $(N=28)$. On multivariate Cox regression analysis, low basal albumin $(P<.01)$ was associated with death, and fever at admission was related to better survival $(P<.01)$. Conclusion. Albumin levels or fever are independent predictors of survival in patients with HIV and disseminated TB. They can serve as indirect markers of immunodeficiency in patients with disseminated TB and AIDS.
\end{abstract}

\section{Introduction}

The advent of highly active antiretroviral therapy (HAART) has changed the natural history of most opportunistic infections and reduced mortality rates in patients with AIDS $[1,2]$. For Mycobacteria tuberculosis (MTB) disease, several studies have demonstrated the protective effect of HAART, in HIV-infected patients [3-6]. However, tuberculosis (TB) is still a potentially fatal complication in these patients $[7$, 8].

Several risk factors are associated with elevated risk of death in patients with TB: advanced age, drug resistance, delayed specific therapy, lack of rifampicin use, inadequate therapy, and HIV infection [9-11]. Few studies addressed this risk in patients with HIV and disseminated TB [12, 13]. The goal of our study was to determine predictors of in-hospital mortality in patients with AIDS and strictly disseminated TB.

\section{Material and Methods}

2.1. Study Population. We performed a retrospective cohort study at Hospital de Clínicas de Porto Alegre, a 740-bed tertiary referral hospital for persons with AIDS in southern Brazil. From 1996 to 2008, all persons who attended as inpatient and had the diagnosis of HIV infection and disseminated TB were included. Patients were selected from the review of microbiology laboratory records, and data were collected by the review of patient's charts. The study was approved by the ethics committee of Hospital de Clínicas de Porto Alegre.

2.2. Definitions. Disseminated tuberculosis was defined as identification of MTB in blood, or bone marrow, or liver biopsy cultures, as suggested by Wang et al. [14]. Only culture positive subjects were included. 
The diagnosis of HIV was made according to current recommendations. For isolation of mycobacteria $[15,16]$, a radiometric system (BACTEC $460 \mathrm{~TB}$-Becton \& Dickinson Diagnostic Instrument Systems, Sparks, MD, USA), and nonradiometric systems (BACTEC 9240-Becton \& Dickinson Diagnostic Instrument Systems, Sparks, MD, USA; and, BacT/Alert 240-bioMérieux, Marcy-Etoile, France) were used. For identification of MTB, NAP (p-nitroalfa-acetylamino-beta-hydroxypropiophenone) test was performed. This test has specificity of $99 \%$ of the identification of Mycobacterium tuberculosis complex [17]. In cases which $N A P$ test could not be done $(N=19,23 \%)$, the identification of species was made by the visualization of the aspect of the colony - presence or absence of cord formation-due to the high sensitivity and specificity of this method [18, 19].

Demographic, clinical, laboratorial, pharmacy, and survival data were collected by chart review. Fever was defined as an axilar temperature $\geq 38.0^{\circ} \mathrm{C}$. Information on concomitant AIDS-related opportunistic infections was collected according to CDC lists [20].

Laboratory data were included, if the sample was collected during the first seven days of hospitalization. CD4 cell count and viral loads were included if they had been determined within six months of hospitalization. Antiretroviral therapy was considered the use of one or more drugs with activity against HIV. Highly active antiretroviral therapy was considered the use of a combination of three or more antiretroviral drugs for HIV therapy. Adequate antituberculous therapy was the use of at least three active drugs against TB for at least seven days, at any time during hospitalization.

2.3. Statistical Analysis. A descriptive analysis of the variables collected from each patient was performed. Continuous variables are shown as median values and the dispersion measurement as range values. The chi-squared test or Fisher's exact test were used for univariate analysis of selected categorical variables. Associations were considered statistically significant when $P$ value was $\leq .05$. Multivariate hazard ratios (HR), along with 95\% confidence intervals $(\mathrm{CI})$, were calculated using the Cox proportional hazards regression model. We included in the final model, the variables with $P$ value $\leq .05$ by univariate analysis. Survival analysis was based on Kaplan-Meier method and comparisons of survival curves were made by the log-rank test. Data were analyzed in SPSS 16.0 version program.

\section{Results}

Eighty-two patients with disseminated tuberculosis were identified. Two patients $(1.5 \%)$ were excluded from the analysis for lack of data. All the patients had clinical or immunological diagnosis of AIDS. The characteristics of patients are shown in Table 1. Forty-nine patients (61\%) were identified by positive blood cultures, twenty-two $(27.5 \%)$ by bone marrow culture, two patients by liver biopsies culture $(2.5 \%)$, five patients $(6.5 \%)$ by blood culture and bone marrow culture, and another two patients $(2.5 \%)$
TABLE 1: Characteristics of the HIV-infected patients with disseminated tuberculosis.

\begin{tabular}{|c|c|}
\hline Median age (range) & $33.0(14-73)$ \\
\hline Men $(\%)$ & $59(69.5)$ \\
\hline \multicolumn{2}{|l|}{ Race } \\
\hline White (\%) & $52(66)$ \\
\hline Black (\%) & $22(28)$ \\
\hline Other $(\%)$ & $5(6)$ \\
\hline \multicolumn{2}{|l|}{ HIV exposure } \\
\hline Sexual (\%) & $31(52.5)$ \\
\hline IV drug use (\%) & $28(48.5)$ \\
\hline Positive Tuberculin Skin Test & $2(5.5)$ \\
\hline Previous $\operatorname{ART}^{1}(\%)$ & $20(25)$ \\
\hline Concomitant opportunistic infection $^{2}(\%)$ & $12(15)$ \\
\hline Median length of hospitalization in days (range) & $24(4-367)$ \\
\hline Median CD4 in cells/mcL (No. of patients) & $39.5(44)$ \\
\hline Median viral load in log/mL (No. of patients) & $4.7(5)$ \\
\hline Anti-HCV positive (\%) & $24(39.5)$ \\
\hline HBsAg positive (\%) & $6(10)$ \\
\hline Appropriate initial TB therapy (\%) & $73(91)$ \\
\hline Median time to TB therapy in days (range) & $7(0-53)$ \\
\hline Median time to death in days (range) & $18.5(2-86)$ \\
\hline \multicolumn{2}{|c|}{$\begin{array}{l}\text { Note. MTB, Mycobacterium tuberculosis; IV, intravenous; ART, antiretroviral } \\
\text { therapy. } \\
{ }^{1} \text { Previous use of ART means previous history of ART. } \\
\text { 2Opportunistic diseases were as follows: cytomegalovirus infection }(N=3) \text {, } \\
\text { Cryptococcus neoformans infection }(N=8) \text {, esophageal candidiasis }(N=6) \text {, } \\
\text { toxoplasmosis }(N=3) \text {, pneumocystis pneumonia }(N=4) \text {, Cryptosporidium } \\
\text { spp }(N=2) \text {. }\end{array}$} \\
\hline
\end{tabular}

by a combination of liver biopsy and bone marrow culture. The median duration of hospitalization was 24 days.

Eighty-six $(N=69)$ percent of the patients had an abnormal X-ray at admission. Sixty-one patients had sputum analysis. Of these, $32.5 \% \quad(N=26)$ were positive for acidfast bacilli.

Fifty-five isolates (68.5\%) were tested for resistance to antituberculous drugs. One isolate was resistant to rifampicin and pirazinamide, other was resistant to isoniazid and streptomycin, and four isolates were resistant to streptomycin. None of the isolates had multidrug-resistant tuberculosis (i.e., resistance to isoniazid and rifampicin). Any drug resistance was not associated with increased patient mortality $(P=.67)$. Rifampicin, isoniazid and pirazinamide, was the initial therapy in $83.5 \%$ of patients $(N=67)$. The median time to therapy was seven days.

In-hospital mortality was 35\% $(N=28)$. Table 2 shows the univariate analysis related to in-hospital death. Low median albumin levels, low hematocrit and hemoglobin levels, high bilirrubin levels, and ICU admission were all related to in-hospital death on univariate analysis. Fever at admission and appropriate initial TB therapy were related to better survival. Considering other clinical presentationsrespiratory symptoms (dyspnea, cough, chest pain), gastrintestinal symptoms (nausea, vomiting, abdominal pain, 
TABLE 2: In-hospital mortality among HIV-infected patients with disseminated tuberculosis.

\begin{tabular}{|c|c|c|c|c|}
\hline \multicolumn{5}{|c|}{ In-hospital death } \\
\hline & Yes & No & Total & $P$ \\
\hline Male & $22(78.5)$ & $37(71)$ & $59(74)$ & .60 \\
\hline Median Age in years (range) & $33(16-73)$ & $33(14-70)$ & $33(N=78)$ & .75 \\
\hline Concomitant opportunistic infection & $3(10.5)$ & $9(17.5)$ & $12(15)$ & .53 \\
\hline ICU admission & $14(50)$ & $4(7.5)$ & $18(22.5)$ & $<.01$ \\
\hline Fever at admission & $17(60.5)$ & $50(96)$ & $67(84)$ & $<.01$ \\
\hline Median alkaline phosphatase (U/L) & 601 & 401 & $426(N=71)$ & .58 \\
\hline (range) & $(62-2539)$ & $(50-4991)$ & & \\
\hline Median ALT (U/L) & 47 & 43 & $43(N=74)$ & .41 \\
\hline (range) & $(10-389)$ & $(9-429)$ & & \\
\hline Median AST (U/L) & 100 & 58 & $69(N=76)$ & .82 \\
\hline (range) & $(18-544)$ & $(12-1575)$ & & \\
\hline Median LDH (U/L) & 730 & 723 & $725(N=71)$ & .91 \\
\hline (range) & $(227-1627)$ & $(172-2329)$ & & \\
\hline Median Bilirrubin (mg/dL) & 1.3 & 0.7 & $0.8(N=72)$ & .02 \\
\hline (range) & $(0.3-8.1)$ & $(0.3-5.2)$ & & \\
\hline Median Hematocrit (\%) & 23 & 27 & $26(N=80)$ & .03 \\
\hline (range) & $(13-34)$ & $(8-43)$ & & \\
\hline Median Hemoglobin (g/dL) & 7.6 & 8.9 & $8.8(N=80)$ & .02 \\
\hline (range) & $(5.5-11.1)$ & $(4.6-13.4)$ & & \\
\hline Median Leukocytes $\left(10^{9} / \mathrm{L}\right)$ & 4.4 & 5.1 & $4.8(N=80)$ & .14 \\
\hline (range) & $(0.5-10.2)$ & $(0.5-31.6)$ & & \\
\hline Median Albumin (g/dL) & 2.2 & 2.7 & $2.6(N=67)$ & $<.01$ \\
\hline (range) & $(1.4-3.1)$ & $(1.7-4.0)$ & & \\
\hline Median Creatinine (mg/dL) & 0.9 & 0.8 & $0.9(N=79)$ & .16 \\
\hline (range) & $(0.2-2.9)$ & $(0.4-1.7)$ & & \\
\hline Median CD4 (cells/mcL) & 36 & 39 & $39(N=44)$ & .92 \\
\hline (range) & $(1-327)$ & $(2-469)$ & & \\
\hline In-hospital HAART ${ }^{1}$ & $6(22.2)$ & $7(14.3)$ & $13(17.1)$ & .53 \\
\hline Appropriate initial TB therapy & $23(82.1)$ & $50(96.2)$ & $73(91.2)$ & .04 \\
\hline Median time to therapy (days) & 6.5 & 7.0 & $7.0(N=70)$ & .54 \\
\hline (range) & $(0.0-49.0)$ & $(0.0-53.0)$ & & \\
\hline
\end{tabular}

Note. Data are no. (\%) of patients, unless otherwise indicated. ICU, Intensive care unit; AFB, acid fast bacilli; ALT, alanine aminotransferase; AST, aspartate aminotransferase; LDH, lactate dehydrogenase; HAART, highly active antiretroviral therapy; TB, tuberculosis.

${ }^{1}$ In-hospital HAART means the prescription of HAART during hospital stay, independent of previous ART use.

diarrhea), adenopathies, weight loss, night sweats, headache or skin lesions-they were not related to death on univariate analysis. Eighteen patients (33.5\%) diagnosed by blood culture died, compared to 10 patients (38.5\%) without mycobacteremia $(P=.80)$.

The median time to initiating therapy in patients with fever was 8 days comparing to four days in patients without fever at admission $(P=.27)$. The median CD4 cell count was 39 cells $/ \mathrm{mcL}$ in patients with fever, and 45 cells $/ \mathrm{mcL}$ in patients without fever at admission $(P=.57)$. Median levels of albumin in patients with fever were $2.6 \mathrm{~g} / \mathrm{dL}$, and $2.3 \mathrm{~g} / \mathrm{dL}$ in patients without fever at admission $(P=.21)$.
As shown in Table 3, the variables included in Cox regression analysis were those with statistical significance on univariate analysis, and concomitant opportunistic infections because of their clinical relation to death. By multivariate analysis, high albumin levels (HR 0.16, CI $0.05-0.56 ; P<.01$ ) and fever at admission (HR 0.18, CI 0.06-0.54; $P<.01$ ) were independently related to better survival. Appropriate initial TB therapy was marginally related to better outcomes (HR 0.25 , CI $0.06-1.06 ; P=.06$ ). Including CD4 counts in the analysis, high albumin levels (HR 0.25, CI 0.07-0.9; $P=.04$ ) and fever at admission (HR 0.20, CI 0.05-0.9; $P=.03$ ) persisted as independent predictors of survival. 


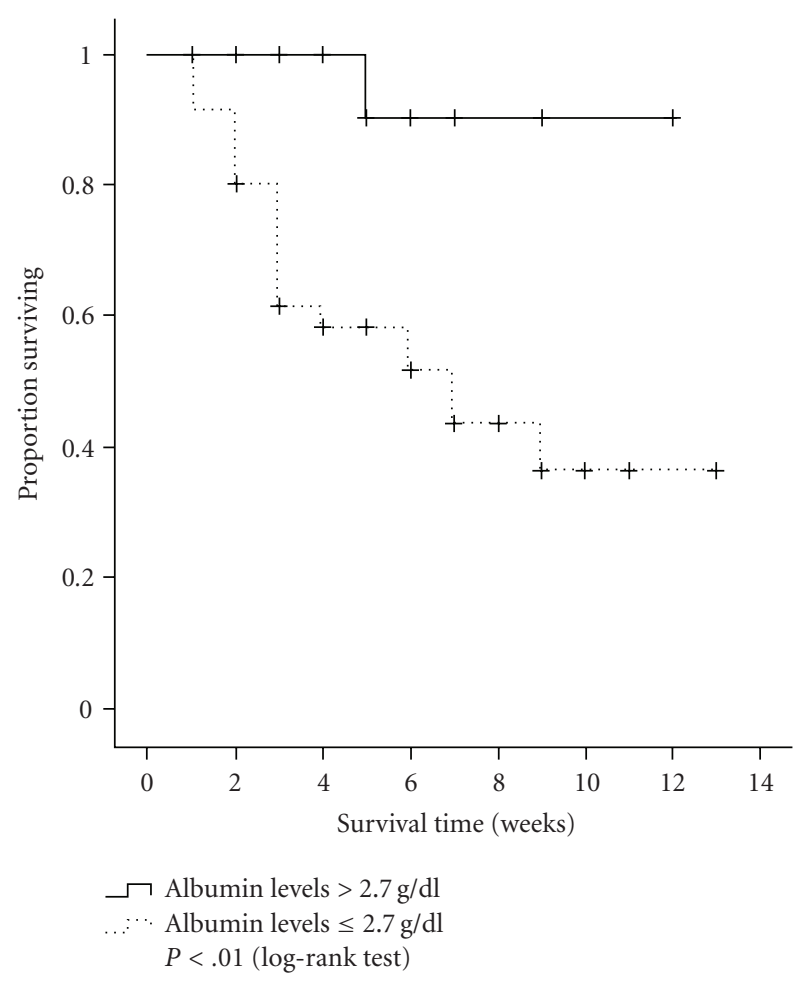

(a)

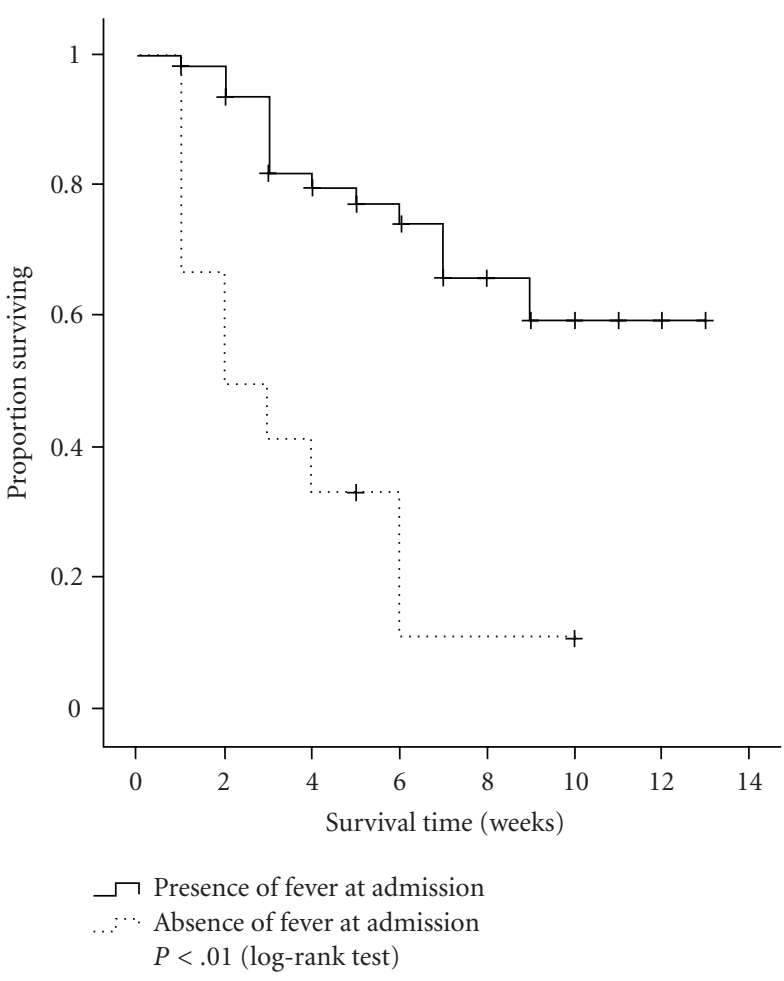

(b)

FIGURE 1: The Kaplan-Meier survival estimates of HIV-infected patients with disseminated TB (a) in relation to basal albumin levels and (b) presence of fever at admission.

Kaplan-Meier curves, as shown in Figure 1, included patients with $\mathrm{TB}$ stratified by albumin levels $(\leq 2.7 \mathrm{~g} / \mathrm{dL}$ versus $>2.7 \mathrm{~g} / \mathrm{dL}$ ) at hospital admission and presence or absence of fever at admission. Patients with levels of albumin $\leq 2.7 \mathrm{~g} / \mathrm{dL}$ had reduced survival rates $(P<.01$; log-rank test $)$. The patients with fever at hospital admission had significant better survival rates $(P<.01$; log-rank test $)$.

\section{Discussion}

Disseminated TB is a predictor of worse prognosis for patients with HIV infection $[13,21]$. In our cohort, we identified albumin levels and fever at admission as additional independent predictors of mortality. These predictors were more strongly associated with mortality than recent CD4 count.

In-hospital mortality in our patients was $35 \%$, similar to prior studies in Thailand and Malawi [22]. Several studies have addressed predictors of mortality in patients with TB. Risk factors for mortality include older age, presence of other comorbid illnesses, emergency department admissions [23]; corticosteroid use [24]; presence of seizures or coma in patients with tuberculous meningitis [25]; multiple organ failure and consolidation on chest radiographs, tuberculousdestroyed lungs, Acute Physiology and Chronic Health Evaluation II scores $\geq 20$, and sepsis in patients who suffered acute respiratory failure $[26,27]$; acute renal failure, need for mechanical ventilation, chronic pancreatitis, sepsis, acute
Table 3: Cox regression analysis. Predictors of in-hospital mortality in HIV-infected patients with disseminated tuberculosis $(N=59)$.

\begin{tabular}{lccc}
\hline & $\begin{array}{c}\text { Hazard Ratio } \\
(\mathrm{HR})\end{array}$ & $\begin{array}{c}\text { Confidence } \\
\text { Interval }\end{array}$ & $P$ \\
\hline Fever at admission & 0.18 & $0.06-0.54$ & $<.01$ \\
High albumin levels & 0.16 & $0.05-0.56$ & $<.01$ \\
Appropriate initial TB therapy & 0.25 & $0.06-1.06$ & .06 \\
High bilirrubin levels & 1.09 & $0.88-1.35$ & .41 \\
Concomitant opportunistic & 0.52 & $0.09-3.06$ & .47 \\
infection & 1.06 & $0.82-1.36$ & .64 \\
Low hemoglobin levels & 0.98 & $0.33-2.57$ & .97 \\
\hline ICU admission & &
\end{tabular}

Note. HR, hazard ratio for hospital death; ICU, intensive care unit; TB, tuberculosis. High or low levels were per $1 \mathrm{~g} / \mathrm{dL}$ change for albumin and hemoglobin and per $1 \mathrm{mg} / \mathrm{dL}$ for bilirrubins.

respiratory distress syndrome, and nosocomial pneumonia in patients admitted to the ICU [28]; None of these studies addressed specifically risk factors for death in patients with disseminated tuberculosis.

Grinsztejn et al. reported that the in-hospital mortality was higher among patients with mycobacterial bacteremia compared to patients without bacteremia, in their study of 42 patients with $M$. avium and M. tuberculosis infection [12]. In our study, the patients had a priori the diagnosis of 
disseminated disease. Mycobacteremia per se was not related to worse prognosis when compared to patients without MTB bacteremia.

Low levels of albumin $(\leq 2.7 \mathrm{mg} / \mathrm{dL})$ during the first seven days in the hospital were an independent predictor of in-hospital death in our patients with disseminated TB. In the EuroSida risk score study and in the study by Sacks et al., body mass index has been implicated with disease progression and death $[29,30]$. Matos and Moreira Lemos reported that serum albumin levels were associated with inhospital death in a 373-person Brazilian TB cohort with 9\% HIV coinfection and without disseminated TB disease [10]. Another study in Israel [25] found that cachexia and lower albumin levels were related to mortality on multivariate analysis. In the Israeli study, 49 (11\%) patients were infected with HIV. Furthermore, hypoalbuminaemia is a marker of severe malnutrition and was a predictor of low CD4 counts in HIV-negative TB patients [31].

Several cytokines have the capacity to induce fever and play an important role in the modulation of the immune response. The list of pyrogenic cytokines includes, among others, interleukin (IL)-1, (TNF)-alpha, IL-6, and IFNgamma [32]. Secretion of these proinflammatory cytokines results in fever and the general constitutional symptoms associated with MTB as a result of the active adaptive cellmediated immune response to MTB. This adaptive response is lead by IFN-gamma secreted by antigen specific T-helper Type 1 (Th1) cells. Several studies have demonstrated that IFN-gamma levels in MTB patients correlate with fever $[33,34]$. Thus, the presence of fever likely represents some capacity to mount a partial immune response to MTB, despite the advanced immunosuppression. Hiraki et al. showed that the pleural fluid concentrations of IL-12, IL-18, and IFN-gamma in tuberculosis patients were correlated to high fever $\left(>38^{\circ} \mathrm{C}\right)[35]$.

Patients with fever at admission had better in-hospital outcomes in our study. Our patients were severely immunossuppressed (median CD4 39.5 cells $/ \mathrm{mcL}$ ), but only 44 patients had CD4 T cells measured. Although fever was not related to CD4 cell count in our sample and we did not evaluate levels of cytokines and IFN-gamma, the presence of fever at admission could indicate the patients with better immune response against MTB and, consequently, predict those with better outcomes.

Considering that most patients $(91.5 \%)$ were adequately treated following standard guidelines, the short median time to initiate therapy after the diagnosis, the good in-hospital compliance to anti-TB medication, and the low level of drug resistance $(3.5 \%)$ to first-line tuberculostatic agents, we can state that our patients seemed to have an aggressive form of disease and/or were diagnosed in their late stage of disseminated tuberculosis.

Our study has the limitations related to a single-site retrospective cohort study. Firstly, the selection bias: we included only patients with positive cultures for TB, possibly excluding patients with less severe disease, in which blood cultures were less likely to be obtained. Moreover, the missing data, related to the retrospective review, reduced the sample size in the multivariate analysis, which could result in overfitting problem (high number of variables included in relation to events) in the Cox regression analysis. Finally, the observational design precludes us to make definite conclusions about specific TB therapy and use of HAART in relation to outcome.

\section{Conclusions}

Disseminated TB was associated with a high in-hospital mortality rate, despite adequate therapy, state-of-the-art diagnostics, and clinical care. For this reason, we emphasize the importance of HIV testing, access to antiretroviral therapy, and screening and treatment of latent tuberculosis infection in order to prevent the disease and mortality in patients with AIDS. Low basal albumin levels were independently related to in-hospital mortality, and fever at admission was related to better prognosis in HIV-infected patients with disseminated TB and AIDS.

\section{Acknowledgment}

The authors would like to thank FIPE (Fundo de Incentivo à Pesquisa-Hospital de Clínicas de Porto Alegre, Brazil) for partial support of this study. All authors contributed equally to this work.

\section{References}

[1] L. R. Fairall, M. O. Bachmann, G. M. C. Louwagie et al., "Effectiveness of antiretroviral treatment in a South African program: a cohort study," Archives of Internal Medicine, vol. 168, no. 1, pp. 86-93, 2008.

[2] J. R. P. Marins, L. F. Jamal, S. Y. Chen et al., "Dramatic improvement in survival among adult Brazilian AIDS patients," AIDS, vol. 17, no. 11, pp. 1675-1682, 2003.

[3] C.-C. Hung, M.-Y. Chen, C.-F. Hsiao, S.-M. Hsieh, W.-H. Sheng, and S.-C. Chang, "Improved outcomes of HIV-1infected adults with tuberculosis in the era of highly active antiretroviral therapy," AIDS, vol. 17, no. 18, pp. 2615-2622, 2003.

[4] A. Miranda, M. Morgan, L. Jamal et al., "Impact of antiretroviral therapy on the incidence of tuberculosis: the Brazilian experience, 1995-2001," PLoS One, vol. 2, no. 9, article e826, 2007.

[5] C. H. Haar, F. G. J. Cobelens, N. A. Kalisvaart, P. J. H. J. Van Gerven, and J. J. Van Der Have, "HIV-related mortality among tuberculosis patients in the Netherlands, 1993-2001," International Journal of Tuberculosis and Lung Disease, vol. 11, no. 9, pp. 1038-1041, 2007.

[6] W. Manosuthi, S. Chottanapand, S. Thongyen, A. Chaovavanich, and S. Sungkanuparph, "Survival rate and risk factors of mortality among HIV/tuberculosis- coinfected patients with and without antiretroviral therapy," Journal of Acquired Immune Deficiency Syndromes, vol. 43, no. 1, pp. 42-46, 2006.

[7] C. A. Schmaltz, F. M. Santanna, S. C. Neves, et al., "Influence of HIV infection on mortality in a cohort of patients treated for tuberculosis in the context of wide access to HAART, in Rio de Janeiro, Brazil," Journal of Acquired Immune Deficiency Syndromes, vol. 52, no. 5, pp. 623-628, 2009.

[8] E. Girardi, C. A. Sabin, A. D. Monferte et al., "Incidence of tuberculosis among HIV-infected patients receiving highly 
active antiretroviral therapy in Europe and North America," Clinical Infectious Diseases, vol. 41, no. 12, pp. 1772-1782, 2005.

[9] C. Teale, J. M. Goldman, and S. B. Pearson, "The association of age with the presentation and outcome of tuberculosis: a five-year survey," Age and Ageing, vol. 22, no. 4, pp. 289-293, 1993.

[10] E. D. Matos and A. C. Moreira Lemos, "Association between serum albumin levels and in-hospital deaths due to tuberculosis," International Journal of Tuberculosis and Lung Disease, vol. 10, no. 12, pp. 1360-1366, 2006.

[11] M. M. Park, A. L. Davis, N. W. Schluger, H. Cohen, and W. N. Rom, "Outcome of MDR-TB patients, 1983-1993," American Journal of Respiratory and Critical Care Medicine, vol. 153, no. 1, pp. 317-324, 1996.

[12] B. Grinsztejn, F. C. O. Fandinho, V. G. Veloso et al., "Mycobacteremia in patients with the acquired immunodeficiency syndrome," Archives of Internal Medicine, vol. 157, no. 20, pp. 2359-2363, 1997.

[13] E. V. Kourbatova, M. K. Leonard Jr., J. Romero, C. Kraft, C. Del Rio, and H. M. Blumberg, "Risk factors for mortality among patients with extrapulmonary tuberculosis at an academic inner-city hospital in the US," European Journal of Epidemiology, vol. 21, no. 9, pp. 715-721, 2006.

[14] J.-Y. Wang, P.-R. Hsueh, S.-K. Wang et al., "Disseminated tuberculosis: a 10-year experience in a medical center," Medicine, vol. 86, no. 1, pp. 39-46, 2007.

[15] T. E. Kiehn and R. Cammarata, "Laboratory diagnosis of mycobacterial infections in patients with acquired immunodeficiency syndrome," Journal of Clinical Microbiology, vol. 24, no. 5, pp. 708-711, 1986.

[16] J. A. Crump, D. C. Tanner, S. Mirrett, C. M. McKnight, and L. B. Reller, "Controlled comparison of BACTEC 13A, MYCO/F LYTIC, BacT/ALERT MB, and ISOLATOR 10 systems for detection of mycobacteremia," Journal of Clinical Microbiology, vol. 41, no. 5, pp. 1987-1990, 2003.

[17] M. A. Morgan, K. A. Doerr, H. O. Hempel, N. L. Goodman, and G. D. Roberts, "Evaluation of the p-Nitro- $\alpha$ Acetylamnino- $\beta$-Hydroxypropiophenone differential test for identification of Mycobacterium tuberculosis complex," Journal of Clinical Microbiology, vol. 21, no. 4, pp. 634-635.

[18] O. Köksalan, M. Aydin, S. Eraslan, and N. Bekiroglu, "Reliability of cord formation in BACTEC 12B/13A media for presumptive identification of Mycobacterium tuberculosis complex in laboratories with a high prevalence of Mycobacterium tuberculosis," European Journal of Clinical Microbiology and Infectious Diseases, vol. 21, no. 4, pp. 314-317, 2002.

[19] F. Zuhre Badak, S. Goksel, R. Sertoz, A. Guzelant, A. Kizirgil, and A. Bilgic, "Cord formation in MB/BacT medium is a reliable criterion for presumptive identification of Mycobacterium tuberculosis complex in laboratories with high prevalence of M. tuberculosis," Journal of Clinical Microbiology, vol. 37, no. 12, pp. 4189-4191, 1999.

[20] CDC, "1993 revised classification system for HIV infection and expanded surveillance case definition for AIDS among adolescents and adults," Morbidity and Mortality Weekly Report, vol. 41, no. RR-17, pp. 4-19, 1992.

[21] C. Whalen, C. R. Horsburgh Jr., D. Hom, C. Lahart, M. Simberkoff, and J. Ellner, "Site of disease and opportunistic infection predict survival in HIV-assosiated truberculosis," AIDS, vol. 11, no. 4, pp. 455-460, 1997.
[22] L. C. McDonald, L. K. Archibald, S. Rheanpumikankit et al., "Unrecognised Mycobacterium tuberculosis bacteraemia among hospital inpatients in less developed countries," The Lancet, vol. 354, no. 9185, pp. 1159-1163, 1999.

[23] N. N. Hansel, B. Merriman, E. F. Haponik, and G. B. Diette, "Hospitalization for tuberculosis in the United States in 2000: predictors of in-hospital mortality," Chest, vol. 126, no. 4, pp. 1079-1086, 2004.

[24] E. Lubart, M. Lidgi, A. Leibovitz, C. Rabinovitz, and R. Segal, "Mortality of patients hospitalized for active tuberculosis in Israel," Israel Medicine Associaton Journal, vol. 9, no. 12, pp. 870-873, 2007.

[25] S. Hosoglu, M. F. Geyik, I. Balik et al., "Predictors of outcome in patients with tuberculous meningitis," International Journal of Tuberculosis and Lung Disease, vol. 6, no. 1, pp. 64-70, 2002.

[26] P. L. Lee, J. S. Jerng, Y. L. Chang et al., "Patient mortality of active pulmonary tuberculosis requiring mechanical ventilation," European Respiratory Journal, vol. 22, no. 1, pp. 141-147, 2003.

[27] Y. J. Ryu, W.-J. Koh, E. H. Kang et al., "Prognostic factors in pulmonary tuberculosis requiring mechanical ventilation for acute respiratory failure," Respirology, vol. 12, no. 3, pp. 406411, 2007.

[28] R. Erbes, K. Oettel, M. Raffenberg, H. Mauch, M. SchmidtIoanas, and H. Lode, "Characteristics and outcome of patients with active pulmonary tuberculosis requiring intensive care," European Respiratory Journal, vol. 27, no. 6, pp. 1223-1228, 2006.

[29] A. Mocroft, B. Ledergerber, K. Zilmer et al., "Short-term clinical disease progression in HIV-1-positive patients taking combination antiretroviral therapy: the EuroSIDA risk-score," AIDS, vol. 21, no. 14, pp. 1867-1875, 2007.

[30] L. V. Sacks and S. Pendle, "Factors related to in-hospital deaths in patients with tuberculosis," Archives of Internal Medicine, vol. 158, no. 17, pp. 1916-1922, 1998.

[31] B. E. Jones, M. M. Oo, E. K. Taikwel et al., "CD4 cell counts in human immunodeficiency virus-negative patients with tuberculosis," Clinical Infectious Diseases, vol. 24, no. 5, pp. 988-991, 1997.

[32] V. Pasquinelli, J. C. Townsend, J. O. Jurado et al., "IFN$\gamma$ production during active tuberculosis is regulated by mechanisms that involve IL-17, SLAM, and CREB," Journal of Infectious Diseases, vol. 199, no. 5, pp. 661-665, 2009.

[33] E. V. D. Vosse, M. A. Hoeve, and T. H. M. Ottenhoff, "Human genetics of intracellular infectious diseases: molecular and cellular immunity against mycobacteria and salmonellae," The Lancet Infectious Diseases, vol. 4, no. 12, pp. 739-749, 2004.

[34] T. C. Y. Tsao, C.-C. Huang, W.-K. Chiou, P.-Y. Yang, M.-J. Hsieh, and K.-C. Tsao, "Levels of interferon- $\gamma$ and interleukin2 receptor- $\alpha$ for bronchoalveolar lavage fluid and serum were correlated with clinical grade and treatment of pulmonary tuberculosis," International Journal of Tuberculosis and Lung Disease, vol. 6, no. 8, pp. 720-727, 2002.

[35] A. Hiraki, K. Aoe, K. Matsuo et al., "Simultaneous measurement of T-helper 1 cytokines in tuberculous pleural effusion," International Journal of Tuberculosis and Lung Disease, vol. 7, no. 12, pp. 1172-1177, 2003. 


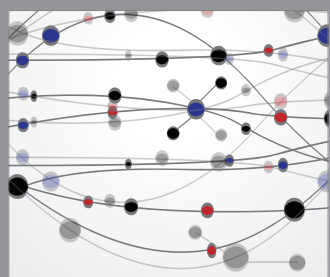

The Scientific World Journal
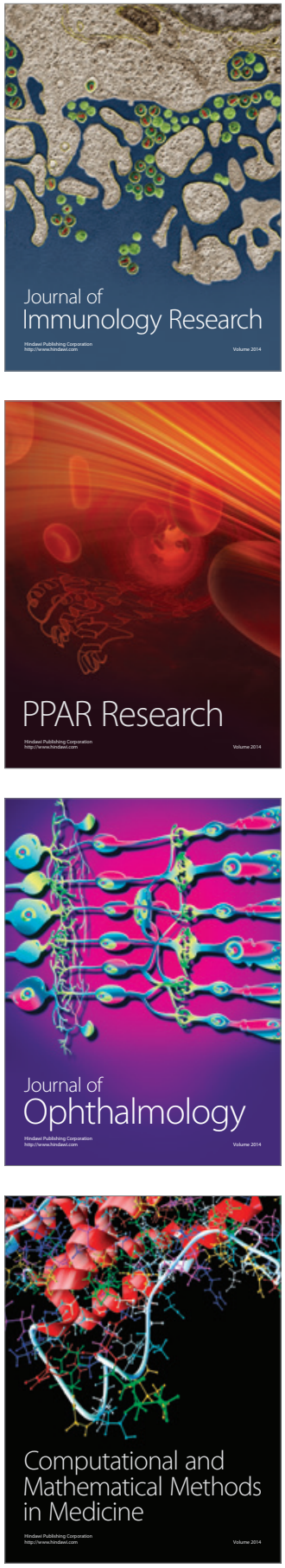

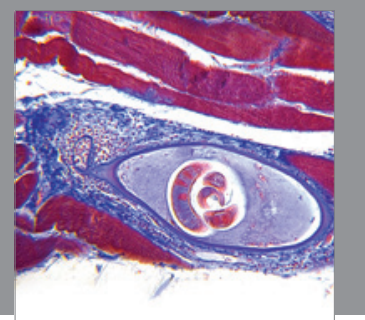

Gastroenterology

Research and Practice
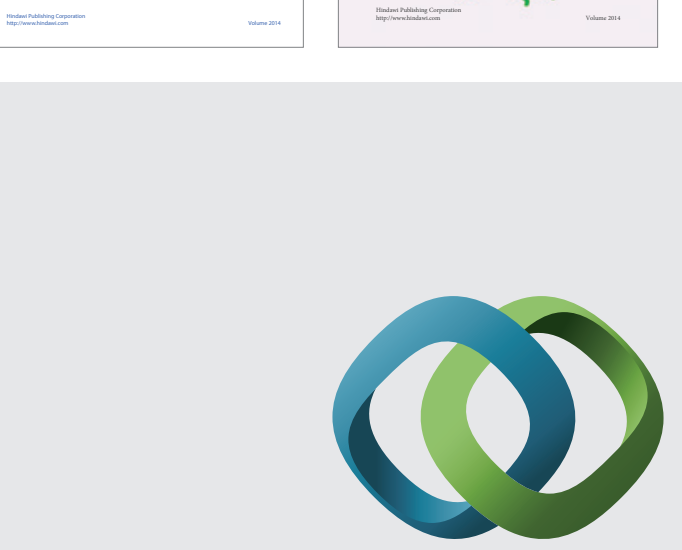

\section{Hindawi}

Submit your manuscripts at

http://www.hindawi.com
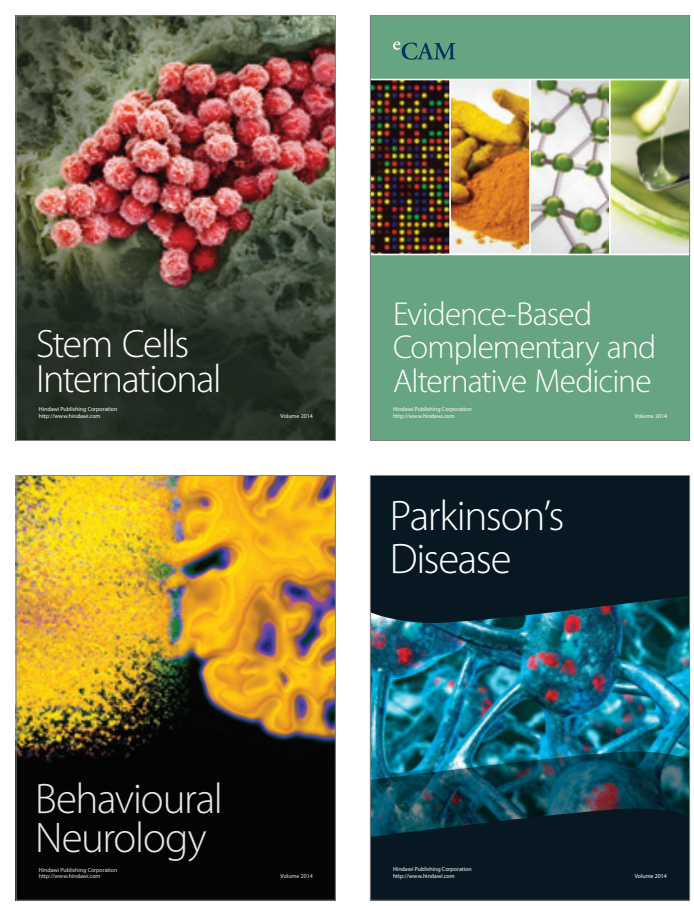

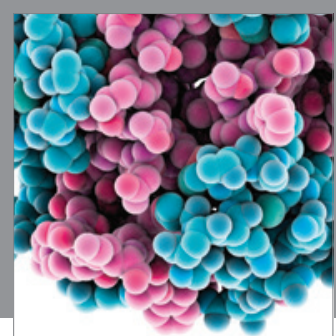

Journal of
Diabetes Research

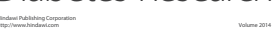

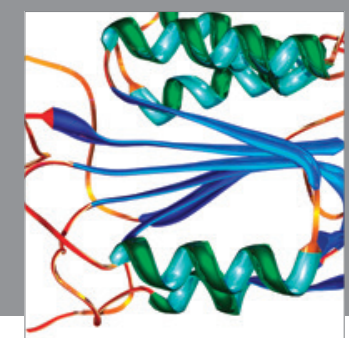

Disease Markers
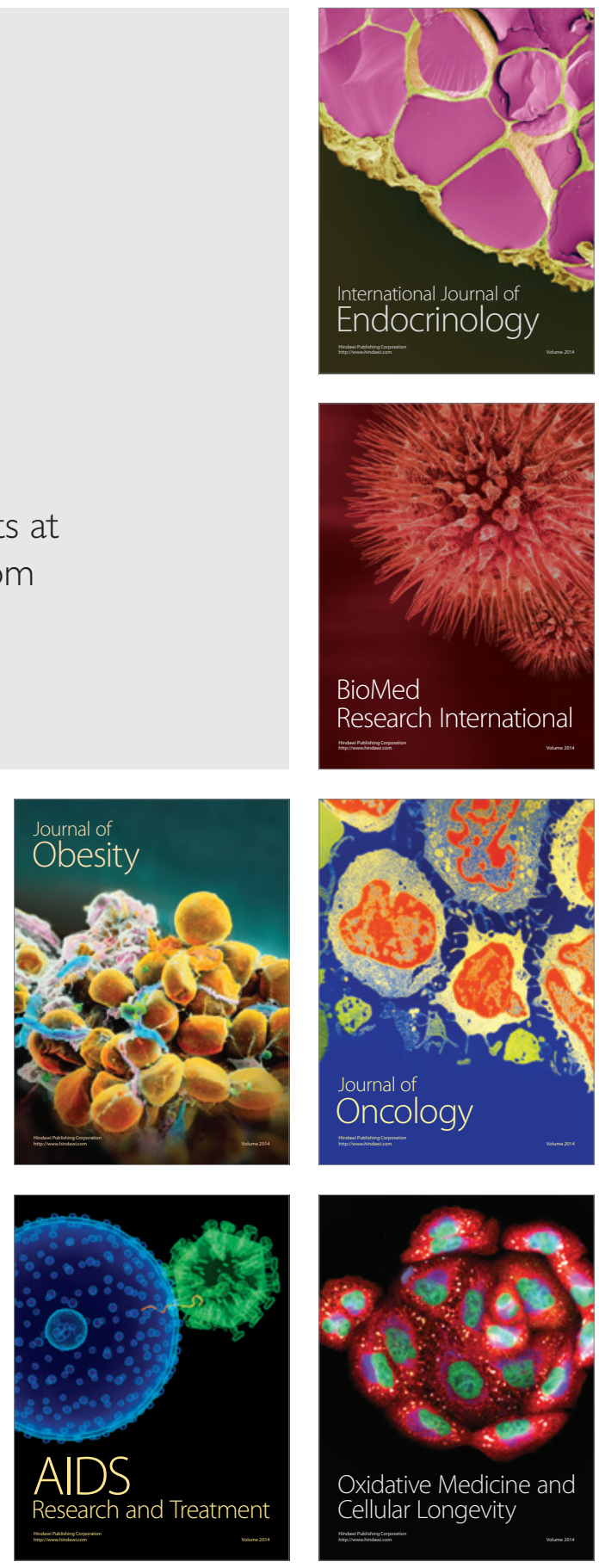